Modern Physics Letters A

(C) World Scientific Publishing Company

\title{
Astrophysical uncertainties on direct detection experiments
}

\author{
Anne M. Green \\ School of Physics and Astronomy, University of Nottingham, University Park, Nottingham, \\ $N G 7$ 2RD, UK \\ anne.green@nottingham.ac.uk
}

\begin{abstract}
Direct detection experiments are poised to detect dark matter in the form of weakly interacting massive particles (WIMPs). The signals expected in these experiments depend on the ultra-local WIMP density and velocity distribution. Firstly we review methods for modelling the dark matter distribution. We then discuss observational determinations of the local dark matter density, circular speed and escape speed and the results of numerical simulations of Milky Way-like dark matter halos. In each case we highlight the uncertainties and assumptions made. We then overview the resulting uncertainties in the signals expected in direct detection experiments, specifically the energy, time and direction dependence of the event rate. Finally we conclude by discussing techniques for handling the astrophysical uncertainties when interpreting data from direct detection experiments.
\end{abstract}

Keywords: dark matter, direct detection experiments

95.35. + d,98.35.Gi,

\section{Introduction}

Weakly Interacting Massive Particles (WIMPs) are a promising dark matter candidate as they are generically produced in the early Universe with roughly the right density. Furthermore supersymmetry (SUSY) provides a well-motivated concrete WIMP candidate in the form of the lightest neutralino (for reviews see e.g. Refs. 1, 2, 3). WIMPs can be directly detected in the lab via their elastic scattering off target nuclei 4 . Numerous direct detection experiments are underway and they are probing the regions of WIMP mass-cross-section parameter space populated by SUSY models (see e.g. Ref. 5).

The realisation that uncertainties in the velocity distribution, $f(\mathbf{v})$, will affect the direct detection signals dates back to some of the earliest direct detection papers written in the 1980s (e.g. Ref. 6). We first discuss the standard halo model and other approaches to modelling the Milky Way halo (Sec. 2). We then discuss what observations and simulations tell us about the dark matter distribution (Secs. 3 and 4 respectively). We then examine the direct detection signals (Sec. 5) and how the astrophysical uncertainties affect these signals (Sec. 6). We conclude in Sec. 7 by discussing strategies for handling the uncertainties, including both 'astrophysics independent' approaches and parameterizing the WIMP speed distribution. 


\section{Halo modelling}

\subsection{Standard halo model}

The steady-state phase space distribution function, $f$, of a collection of collisionless particles is given by the solution of the collisionless Boltzmann equation:

$$
\frac{\mathrm{d} f}{\mathrm{~d} t}=0
$$

In Cartesian coordinates this becomes

$$
\frac{\partial f}{\partial t}+\mathbf{v} \cdot \frac{\partial f}{\partial \mathbf{x}}-\frac{\partial \Phi}{\partial \mathbf{x}} \frac{\partial f}{\partial \mathbf{v}}=0
$$

where $\Phi$ is the potential.

The standard halo model (SHM) is an isotropic, isothermal sphere with density profile $\rho(r) \propto r^{-2}$. In this case the solution of the collisionless Boltzmann equation is a so-called Maxwellian velocity distribution, given by

$$
f(\mathbf{v})=N \exp \left(-\frac{3|\mathbf{v}|^{2}}{2 \sigma^{2}}\right),
$$

where $N$ is a normalisation constant. The isothermal sphere has a flat rotation curve at large radii and the velocity dispersion is related to the asymptotic value of the circular speed (the speed with which objects on circular orbits orbit the Galactic centre) $v_{\mathrm{c}, \infty}=\sqrt{2 / 3} \sigma$. It is usually assumed that the rotation curve has already reached its asymptotic value at the Solar radius, $r=R_{0}$, so that $\sigma=\sqrt{3 / 2} v_{\mathrm{c}}$ where $v_{\mathrm{c}} \equiv v_{\mathrm{c}}\left(R_{0}\right)$ is the local circular speed. In the SHM the peak speed $v_{0}$ and the circular speed are identical, $v_{\mathrm{c}}=v_{0}$, and these parameters are often used interchangeably. However this is not the case in general, for instance for a NFW 7 density profile $v_{\mathrm{c}}=0.88 v_{0}$ at $r=r_{\mathrm{s}} / 2$ (where the scale radius, $r_{\mathrm{s}}$, is the radius at which the logarithmic slope of the density profile is equal to -2 ) 8 .

The density distribution of the SHM is formally infinite and hence the velocity distribution extends to infinity too. In reality the Milky Way halo is finite, and particles with speeds greater than the escape speed, $v_{\text {esc }}(r)=\sqrt{2|\Phi(r)|}$, will not be gravitationally bound to the MW. This is often addressed by simply truncating the velocity distribution at the measured local escape speed, $v_{\text {esc }} \equiv v_{\text {esc }}\left(R_{0}\right)$, so that $f(\mathbf{v})$ is given by eq. (3) for $|\mathbf{v}|<v_{\text {esc }}$ and $f(\mathbf{v})=0$ for $|\mathbf{v}| \geq v_{\text {esc }}$. This sharp truncation is clearly unphysical. An alternative, but still ad hoc, approach is to make the truncation smooth:

$$
f(\mathbf{v})= \begin{cases}N\left[\exp \left(-\frac{3|\mathbf{v}|^{2}}{2 \sigma^{2}}\right) \exp \left(-\frac{3 v_{\mathrm{esc}}^{2}}{2 \sigma^{2}}\right)\right], & |\mathbf{v}|<v_{\mathrm{esc}} \\ 0, & |\mathbf{v}| \geq v_{\mathrm{esc}}\end{cases}
$$

Another approach, used in the King model/lowered isothermal sphere, is to modify the SHM distribution function so that it becomes zero for large energies 9 .

The standard parameter values used for the SHM are a local density $\rho_{0} \equiv$ $\rho\left(R_{0}\right)=0.3 \mathrm{GeV} \mathrm{cm}^{-3}$, a local circular speed $v_{\mathrm{c}}=220 \mathrm{~km} \mathrm{~s}^{-1} 10$, and a local escape 
speed $v_{\text {esc }}=544 \mathrm{~km} \mathrm{~s}^{-1}$ 11. We will discuss the determination of these parameters and the associated uncertainties in Sec. 3 .

\subsection{Non-standard halo models}

For spherical, isotropic systems there is a unique relationship between the density profile and the distribution function known as the Eddington equation (see e.g. Ref. 9, 12):

$$
f(\epsilon)=\frac{1}{\sqrt{8} \pi^{2}}\left[\int_{0}^{\epsilon} \frac{\mathrm{d} \Psi}{\sqrt{\epsilon-\Psi}} \frac{\mathrm{d}^{2} \rho}{\mathrm{d} \Psi^{2}}+\frac{1}{\sqrt{\epsilon}}\left(\frac{\mathrm{d} \rho}{\mathrm{d} \Psi}\right)_{\Psi=0}\right],
$$

where $\Psi(r)=-\Phi(r)+\Phi(r=\infty)$ and $\epsilon=-E_{\text {kin }}+\Psi(r)$, where $E_{\text {kin }}$ is the kinetic energy. The Eddington equation has been used to calculate the speed distribution for a range of spherically symmetric density profiles 12 13/14/15. Ref. 15 found, using a Bayesian analysis incorporating various dynamical constraints, speed distributions that are not too dissimilar to the standard Maxwellian (when the same value of the circular speed is used). Ref. 16 used the Eddington equation to motivate a phenomenological form for $f(|\mathbf{v}|)$ which matches the high speed tail of the speed distributions found in simulated halos (see Sec. 4).

The Osipkov-Merritt models 17 assume that the distribution function depends on the energy, $\epsilon$, and angular momentum, $L$, only via a single parameter, $Q$,

$$
Q=\epsilon-\frac{L^{2}}{2 r_{a}^{2}},
$$

where the constant $r_{a}$ is the anisotropy radius. In this model the velocity anisotropy

$$
\beta=1-\frac{\sigma_{\theta}^{2}+\sigma_{\phi}^{2}}{2 \sigma_{r}^{2}}
$$

where $\sigma_{\theta, \phi, r}$ are the velocity dispersions in the $r, \theta$ and $\phi$ directions, has a particular, radially dependent, form:

$$
\beta=\frac{1}{1+\left(r_{a}^{2} / r^{2}\right)},
$$

and the distribution function can be calculated from a modified version of the Eddington equation (see e.g. Refs. 9, 12). In these models the peak of the speed distribution becomes narrower and there is an excess of high speed particles (e.g. Ref. [18).

In general there is no unique relationship between the density profile and the velocity distribution for triaxial and/or anisotropic systems. In these cases a common approach is to use the Jeans equations to calculate the lower order moments of the distribution function. Multiplying the collisionless Boltzmann equation, eq. (2), by

one of the velocity components and integrating gives the Jeans equations, which in Cartesian coordinates take the form

$$
\frac{\partial\left(\rho \bar{v}_{j}\right)}{\partial t}+\frac{\partial\left(\rho \overline{v_{j} v_{i}}\right)}{\partial x_{i}}+\rho \frac{\partial \Phi}{\partial x_{j}}=0
$$


We now have three equations (corresponding to $j=1,2,3$ ) for six unknowns $\left(\overline{v_{1}^{2}}, \overline{v_{2}^{2}}, \overline{v_{3}^{2}}, \overline{v_{1} v_{2}}, \overline{v_{2} v_{3}}, \overline{v_{1} v_{3}}\right)$. To make further progress it is therefore necessary to make assumptions about the alignment of the velocity ellipsoid, i.e. to choose coordinates such that $\overline{v_{i} v_{j}}=0$ for $i \neq j$. The velocity distribution is then approximated by a multivariate gaussian in these coordinates:

$$
f(\mathbf{v}) \propto \exp \left(-\frac{v_{1}^{2}}{2 \sigma_{1}^{2}}-\frac{v_{2}^{2}}{2 \sigma_{2}^{2}}-\frac{v_{3}^{2}}{2 \sigma_{3}^{2}}\right),
$$

where $\sigma_{i}^{2} \equiv \overline{v_{i}^{2}}$.

Evans, Carollo and de Zeeuw 19 studied the logarithmic ellipsoidal model, the simplest triaxial generalisation of the isothermal sphere. They argue that aligning the velocity ellipsoid with conical coordinates is physically well motivated. In the planes of the halo, conical coordinates are locally equivalent to cylindrical polar coordinates. Hence, in this model, the velocity distribution can be approximated by a multi-variate gaussian in cylindrical polar coordinates, with velocity dispersions depending on the shape of the halo, the velocity anisotropy and also location within the halo. Both the width of the speed distribution and the peak speed can change (e.g. Refs. 19, 18).

Analytic models inevitably make assumptions (regarding the shape and anisotropy of the halo, and their radial dependence) which are almost certainly not completely valid. The relationship between the observed properties of dark matter halos and the velocity distribution is non-trivial; models with the same bulk properties (e.g. shape and local velocity anisotropy) can have velocity distributions with very different forms. Furthermore all analytic approaches to halo modelling rely on the assumption that the phase space distribution function has reached a steady state. To some extent analytic halo models have been superseded by results from numerical simulations. It should be emphasized (see Sec. 4.2 and 4.1) that velocity distributions from numerical simulations also involve approximations and extrapolations.

\section{Observations}

\subsection{Density}

The event rate is directly proportional to the local density, $\rho_{0}$. The standard value used is $\rho_{0}=0.3 \mathrm{GeV} \mathrm{cm}{ }^{-3}=0.008 M_{\odot} \mathrm{pc}^{-3}=5 \times 10^{-25} \mathrm{~g} \mathrm{~cm}^{-3}$. As discussed in Ref. 20, the origin of the use of this particular value, rather than say $\rho_{0}=$ $0.4 \mathrm{GeV} \mathrm{cm}^{-3}$, is unclear.

The local density is calculated via mass modelling of the Milky Way (MW). This involves constructing a model of the MW (including its luminous components) and then finding the range of values of the local density that are consistent with all observational data (including, for instance, rotation curve measurements, velocity dispersions of halo stars, local surface mass density, total mass) 2122. As emphasised in Ref. 22 the shape of the MW halo has a crucial effect on the local density 
extracted. For a fixed circular speed, in a flattened halo the local density, in the Galactic plane, is higher.

This work has recently been updated to include new data sets, models for the MW halo motivated by numerical simulations, and, in some cases, Bayesian statistical techniques. Ref. 23, assuming a spherical halo with a cuspy density profile $\left(\rho(r) \propto r^{-\alpha}\right.$ as $\left.r \rightarrow 0\right)$ found $\rho_{0}=0.30 \pm 0.05 \mathrm{GeV} \mathrm{cm}^{-3}$, while Ref. 24, assuming spherically symmetric NFW 7 and Einasto $25 \mid 26$ profiles for the MW halo found, $\rho_{0}=0.39 \pm 0.03 \mathrm{GeV} \mathrm{cm}^{-3}$. While these determinations have $\sim 10 \%$ errors, they differ from each other by significantly more than this. This suggests that the systematic errors are bigger than the statistical errors. Indeed Ref. 27 finds, considering a range of density profiles including cored profiles $(\rho(r) \sim$ const as $r \rightarrow 0)$, values in the range $\rho_{0}=0.2-0.4 \mathrm{GeV} \mathrm{cm}^{-3}$.

Other recent work has investigated 'model independent' techniques, which don't involve global mass-modelling of the galaxy, and hence have smaller hidden systematic errors. Ref. 20 proposed using the equation of centrifugal equilibrium and subtracting the contribution of the stellar component of the MW. The resulting determination of the local density is $\rho_{0}=0.43 \pm 0.11 \pm 0.10 \mathrm{GeV} \mathrm{cm}^{-3}$, where the uncertainties come from the uncertainty in the slope of the circular speed at the Solar radius and the ratio of the Solar radius to the length scale of the thin stellar disk. Ref. 28 introduced a method which involves solving the Jeans and Poisson equations with minimal assumptions. Using Hipparcos data they find $\rho_{0}=0.11_{-0.27}^{+0.34} \mathrm{GeV} \mathrm{cm}^{-3}$, at $90 \%$ confidence, assuming the stellar tracer populations are isothermal and $\rho_{0}=1.25_{-0.34}^{+0.30} \mathrm{GeV} \mathrm{cm}^{-3}$ if they have a non-isothermal profile. An accurate determination of the vertical dispersion profile of the tracer population is therefore required to make an unbiased estimate of the local density using this method.

Ref. 29 used a high-resolution simulation of a Milky Way like galaxy, including baryons 30 , to investigate the effect of halo shape on the local density. Specifically they examined how the local density varies from the density averaged in a spherical shell, as determined by observations. They find that the density within the stellar disk, at a distance $R_{0}=8 \mathrm{kpc}$ from the centre, is a factor of between 1.01 and 1.41 , with an average of 1.21 , larger than the value averaged in a spherical shell.

\subsection{Circular speed}

The local circular speed, $v_{\mathrm{c}} \equiv v_{\mathrm{c}}\left(R_{0}\right)$, is an important quantity to determine. It appears in the Galilean transformation of the velocity distribution into the lab frame (see Sec. 5). It is also related to the radial component of the velocity dispersion, $\sigma_{r}$, by one of the Jeans equations (e.g. Ref. 9):

$$
\frac{1}{\rho} \frac{\mathrm{d}\left(\rho \sigma_{r}^{2}\right)}{\mathrm{d} r}+2 \frac{\beta \sigma_{r}^{2}}{r}=-\frac{v_{\mathrm{c}}^{2}}{r}
$$

where the anisotropy parameter $\beta$ is defined in eq. (7). The SHM has $\rho(r) \propto r^{-2}$ and the the velocity distribution is isotropic $\left(\sigma_{r}=\sigma_{\theta}=\sigma_{\phi}\right.$ so that $\left.\beta=0\right)$ and 
independent of radius, so that $\sigma_{\mathrm{r}}=v_{\mathrm{c}} / \sqrt{2}$.

The standard value of $v_{\mathrm{c}}$ of $220 \mathrm{~km} \mathrm{~s}^{-1}$ dates back to a $1980 \mathrm{~s}$ review of the Galactic constants 10 and was found by taking an average of the values found from a wide range of different analyses. Note that the ratio $v_{\mathrm{c}} / R_{0}$ is better determined than either $v_{\mathrm{c}}$ or $R_{0}$ individually (e.g. Refs. 31, 32).

A recent analysis using measurements of the motions of Galactic masers, found a significantly higher value, $v_{\mathrm{c}}=(254 \pm 16) \mathrm{km} \mathrm{s}^{-1}$, 31. Ref. 33 reanalyzed the data using a more general model for the maser velocity distribution (including allowing a non-zero velocity dispersion tensor) and found that the maser data places only a relatively weak constraint on $v_{\mathrm{c}}$. When combined with other measurements (from the proper motion of Sgr $\mathrm{A}^{\star}$, and the orbit of the GD-1 stellar stream), they found $v_{\mathrm{c}}=(236 \pm 11) \mathrm{km} \mathrm{s}^{-1}$, assuming a flat rotation curve. Meanwhile Ref. 32 found the value of $v_{\mathrm{c}}$ determined from the maser data depends strongly on the MW model used. Using a range of models for the rotation curve, including a power-law with free slope, they found values in the range $v_{\mathrm{c}}=(200 \pm 20) \mathrm{km} \mathrm{s}^{-1}$ to $v_{\mathrm{c}}=(279 \pm 33) \mathrm{km} \mathrm{s}^{-1}$. This illustrates that, as in the case of the local density, systematic, modelling errors are important.

\subsection{Escape speed}

The escape speed is the speed required to escape the local gravitational field of the MW, $v_{\text {esc }}(r)=\sqrt{2|\Phi(r)|}$. The local escape speed, $v_{\text {esc }} \equiv v_{\text {esc }}\left(R_{0}\right)$, is estimated from the speeds of high velocity stars. To do this it is necessary to parameterise the shape of the high speed tail of the velocity distribution. Assuming that the velocities are isotropic and the Jeans theorem applies, the asymptotic form of the velocity distribution can be written as 3435.

$$
f(|\mathbf{v}|) \propto \begin{cases}\left(v_{\mathrm{esc}}^{2}-|\mathbf{v}|^{2}\right)^{k}=\left[\left(v_{\mathrm{esc}}-|\mathbf{v}|\right)\left(v_{\mathrm{esc}}+|\mathbf{v}|\right)\right]^{k}, & |\mathbf{v}|<v_{\mathrm{esc}} \\ 0, & |\mathbf{v}| \geq v_{\mathrm{esc}}\end{cases}
$$

Traditionally a value $v_{\text {esc }}=650 \mathrm{~km} \mathrm{~s}^{-1}$, corresponding to the upper $90 \%$ confidence limit from Ref. 34, has been used.

Ref. 11 has updated these measurements, using additional data from the RAVE survey and using a prior on $k, k \in[2.7,4.7]$ (motivated by analysis of the speed distributions of stellar particles in simulated halos). They find that the escape speed lies in the range $498 \mathrm{~km} \mathrm{~s}^{-1}<v_{\text {esc }}<608 \mathrm{~km} \mathrm{~s}^{-1}$ at $90 \%$ confidence, with a median likelihood of $v_{\text {esc }}=544 \mathrm{~km} \mathrm{~s}^{-1}$.

\section{Simulations}

A number of high resolution, dark matter only, simulations of the formation of Milky Way-like halos in a cosmological context have been carried out (e.g. Aquarius 36, GHALO 37 and Via Lactea 38). The velocity distributions of these halos deviate systematically from a multivariate Gaussian 39|40|418. There are more low speed 
particles and the peak in the distribution is lower and flatter in shape (i.e. the distribution is platykurtic) a. Several fitting functions have been considered; a Tsallis distribution (which arises from non-extensive statistical mechanics) 4243 and a modified Maxwellian $40 \mid 8$

$$
\begin{aligned}
& f\left(v_{\mathrm{r}}\right)=\frac{1}{N_{\mathrm{r}}} \exp \left[-\left(\frac{v_{\mathrm{r}}^{2}}{\bar{v}_{\mathrm{r}}^{2}}\right)^{\alpha_{\mathrm{r}}}\right], \\
& f\left(v_{\mathrm{t}}\right)=\frac{v_{\mathrm{t}}}{N_{\mathrm{t}}} \exp \left[-\left(\frac{v_{\mathrm{t}}^{2}}{\bar{v}_{\mathrm{t}}^{2}}\right)^{\alpha_{\mathrm{t}}}\right],
\end{aligned}
$$

where $v_{\mathrm{r}}$ and $v_{\mathrm{t}}=\sqrt{v_{\theta}^{2}+v_{\phi}^{2}}$ are the radial and tangential components of the velocity, $N_{\mathrm{r} / \mathrm{t}}$ are normalisation factors and $\alpha_{r, t}$ are free-parameters which parameterise the deviations from a Maxwellian distribution. The most likely speed deviates from the circular speed: for VL2 and GHALO $v_{\mathrm{c}} / v_{0} \approx 0.85$ and 0.86 respectively $[8$.

The velocity distributions also have stochastic features at high speeds. There are broad bumps which vary from halo to halo, but are independent of position within a given halo and are thought to reflect the formation history of the halo $\underline{418}$. Kuhlen et al. $\frac{8}{}$ also find narrow spikes in some locations, corresponding to tidal streams.

Ref. 16 presented an ansatz for the velocity distribution

$$
f(|\mathbf{v}|) \propto\left[\exp \left(\frac{v_{\mathrm{esc}}^{2}-|\mathbf{v}|^{2}}{k v_{0}^{2}}\right)-1\right]^{k} \Theta\left(v_{\mathrm{esc}}-|\mathbf{v}|\right)
$$

which fits the high speed tail of the smooth component of the speed distributions found in simulations. The shape of the high speed tail of the distribution is determined by the slope of the density profile at large radii. Using the Eddington equation the parameter $k$ can be related to the outer slope of the density profile, $\gamma,\left(\rho(r) \propto r^{-\gamma}\right.$ for large $r$ ), by $k=\gamma-3 / 2$ for $\gamma>3 \underline{35}$. As $\gamma \rightarrow 3$ (the value corresponding to the NFW profile ${ }^{7}$ ) the calculation breaks down, and numerical fits to eq. (15) find $k \approx 2$. More generally for outer slopes in the range $\gamma \sim 3-5, k$ lies in the range $k=[1.5-3.5]$. Eq. (15) with $k$ in this range provides a good fit to the high speed tails of the speed distributions found in simulation and has less high speed particles than the tail of the standard Maxwellian distribution with a sharp cut-off. Note, however, that eq. (15) does not match the low and moderate speed behaviour of the simulation speed distributions, possibly due to the assumption of isotropy 16 .

\subsection{Effects of baryons}

The simulations discussed above contain dark matter only, while baryons dominate in the inner regions of the Milky Way. Simulating baryonic physics is extremely difficult, and producing galaxies whose detailed properties match those of real galaxies

a Note, however, that the deviation is smaller in the lab frame than in the Milky Way rest frame 8 . 
is a major challenge. Some recent simulations have found that late merging subhalos are preferentially dragged towards the disc by dynamical friction, where they are destroyed leading to the formation of a co-rotating dark disc (DD) $44 \mid 45146$. Ref. 47 modelled the DD velocity distribution as a gaussian with isotropic dispersion, $\sigma_{\mathrm{DD}}=50 \mathrm{~km} \mathrm{~s}^{-1}$ and lag $v_{\text {lag }}=50 \mathrm{~km} \mathrm{~s}^{-1}$, matching (roughly) the kinematics of the Milky Way's stellar thick disc and considered DD densities in the range $0.5<\left(\rho_{\mathrm{DD}} / \rho_{\mathrm{H}}\right)<2$, where $\rho_{\mathrm{H}}$ is the local halo density.

The properties (and even existence) of the dark disc are highly uncertain. Ref. 48 argues that to be consistent with the observed morphological and kinematic properties of the Milky Way's thick disc, the Milky Way's merger history must be quiescent compared with typical $\Lambda$ CDM merger histories, and hence the DD density must be relatively small, $\rho_{\mathrm{DD}}<0.2 \rho_{\mathrm{H}}$. The total (halo + disc) local density can be probed by the kinematics of stars (e.g. Ref. 49). There is no evidence for a dark matter disc (i.e. the data are well fit without a dark disc) and a thick, dense, $\left(\rho_{\mathrm{DD}} / \rho_{\mathrm{H}}\right)>0.5$, dark disc is excluded 50 . Refs. 48 and 51 also argue that the DD velocity dispersion is likely to be substantially larger than that of the stellar thick disc.

Baryonic physics will also affect the halo speed distribution. For instance gas cooling makes halos more spherical (e.g. Ref.52). The local speed distribution found in Ref. 46 is well fit by a Tsallis distribution, but appears to deviate less from the standard Maxwellian than the distributions found in dark matter only simulations.

\subsection{Ultra-local structure}

A further caution is that the scales resolved by simulations are many orders of magnitude larger than those relevant for direct detection experiments. The Earth moves at $\sim 200 \mathrm{~km} \mathrm{~s}^{-1} \sim 0.1 \mathrm{mpc} \mathrm{yr}^{-1}$, therefore direct detection experiments probe the dark matter on sub mpc scales, while the numerical simulations discussed above have gravitational softening of order $10 \mathrm{pc}$.

Vogelsberger and White have developed a new technique to follow the finegrained phase space distribution in simulations as it stretches and folds under the action of gravity 53. They find that the median density of the resulting streams is of order $10^{-7}$ the local halo density. Schneider et al. $\frac{54}{5}$ have studied the evolution of the first, and smallest, roughly Earth mass $55|56| 57 / 58$, microhalos to form in the Universe. They find that tidal disruption and encounters with stars produce tidal streams with average density $\sim 10^{-4}$ the local halo density. These results (see also Ref. (59) suggest that the ultra-local dark matter density and velocity distribution should not be drastically different to those on the scales resolved by simulations. The ultra-local velocity distribution may, however, contain some features or fine-grained structure $60|61| 62|63| 64$. 


\section{Differential event rate and signals}

The differential event rate for elastic scattering, assuming spin-independent coupling with identical couplings to the proton and neutron, is given by (e.g. Refs. 1, 65):

$$
\frac{\mathrm{d} R}{\mathrm{~d} E}(E, t)=\frac{\sigma_{\mathrm{p}} \rho_{0}}{2 \mu_{\mathrm{p} \chi}^{2} m_{\chi}} A^{2} F^{2}(E) \int_{|\mathbf{v}| \geq v_{\min }} \frac{f(\mathbf{v}, t)}{v} \mathrm{~d}^{3} \mathbf{v}
$$

where $\rho_{0}$ is the local WIMP density, $f(\mathbf{v}, t)$ the WIMP velocity distribution in the lab frame, $\sigma_{\mathrm{p}}$ the WIMP scattering cross section on the proton, $\mu_{\mathrm{p} \chi}=$ $\left(m_{\mathrm{p}} m_{\chi}\right) /\left(m_{\mathrm{p}}+m_{\chi}\right)$ the WIMP-proton reduced mass, $A$ and $F(E)$ the mass number and form factor of the target nuclei respectively and $E$ is the recoil energy. The lower limit of the integral, $v_{\min }$, is the minimum WIMP speed that can cause a recoil of energy $E$ :

$$
v_{\min }=\left(\frac{E m_{A}}{2 \mu_{\mathrm{A} \chi}^{2}}\right)^{1 / 2},
$$

where $m_{A}$ is the atomic mass of the detector nuclei and $\mu_{\mathrm{A} \chi}$ the WIMP-nucleon reduced mass. The event rate for inelastic scattering of WIMPs 66 also depends on the WIMP density and velocity distribution. However, due to the altered kinematics the relationship between $v_{\min }$ and $E$ is changed, and hence the effects of changes in the velocity distribution are different $67 / 8 \mid 51 / 68$.

The WIMP speed distribution must be transformed from the Galactic rest frame to the lab frame. This is done by carrying out, a time dependent, Galilean transformation: $\mathbf{v} \rightarrow \tilde{\mathbf{v}}=\mathbf{v}+\mathbf{v}_{e}(t) \mathrm{b}$. The Earth's motion relative to the Galactic rest frame, $\mathbf{v}_{e}(t)$, is made up of three components: the motion of the Local Standard of Rest (LSR), $\mathbf{v}_{\mathrm{LSR}}$, the Sun's peculiar motion with respect to the LSR, $\mathbf{v}_{\odot}^{\mathrm{p}}$, and the Earth's orbit about the Sun, $\mathbf{v}_{e}^{\text {orb }}$. The motion of the LSR is defined as $\mathbf{v}_{\mathrm{LSR}}=\left(0, v_{\mathrm{c}}, 0\right)$, where $v_{\mathrm{c}}$ is the local circular speed (see Sec. 3.2 for a discussion of recent determinations). The most recent determination of the Sun's motion with respect to the LSR, taking into account the effects of the metallicity gradient in the disc, finds $\mathbf{v}_{\odot}^{\mathrm{p}}=\left(U_{\odot}, V_{\odot}, W_{\odot}\right)=(11.1,12.2,7.3) \mathrm{km} \mathrm{s}^{-1}[1]$ in Galactic co-ordinates (where $U$ points towards the Galactic center, $V$ is the direction of Galactic rotation and $W$ towards the North Galactic Pole). Note that the value of $V_{\odot}$ is significantly $\left(\sim 7 \mathrm{~km} \mathrm{~s}^{-1}\right)$ larger than previously found $\frac{72}{32}$. The new larger value is also supported by the analysis of the motions of masers 32 . Accurate expressions for the Earth's orbit can be found in Ref. 73. Simpler expressions, which are acceptable for most practical purposes, can be found in Refs. 74, 75.

The differential event rate, eq. (16), depends on the target nuclei mass, the (a priori unknown) WIMP mass and the integral of the velocity distribution. It is

\footnotetext{
${ }^{\mathrm{b}}$ Formally gravitational focusing by the Sun should be taken into account $69 \sqrt{70}$, however the resulting modulation in the differential event rate is small and only detectable with a very large number of events 69 .
} 
therefore useful to rewrite eq. (16) as

$$
\frac{\mathrm{d} R}{\mathrm{~d} E}(E, t)=C_{\chi, \mathrm{A}} \rho_{0} g\left(v_{\min }, t\right),
$$

where

$$
g\left(v_{\min }, t\right)=\int_{|\mathbf{v}| \geq v_{\min }} \frac{f(\mathbf{v}, t)}{v} \mathrm{~d}^{3} \mathbf{v}
$$

and the prefactor

$$
C_{\chi, \mathrm{A}}=\frac{\sigma_{\mathrm{p}}}{2 \mu_{\mathrm{p} \chi}^{2} m_{\chi}} A^{2} F^{2}(E),
$$

contains the WIMP and target dependent terms and is independent of the astrophysical WIMP distribution.

We will now discuss the energy (Sec. [5.1), time (Sec. [5.2) and direction (Sec. [5.3) dependence of the differential event rate. In order to make concrete statements we will for now assume the standard Maxwellian velocity distribution, eq. (3). In Sec.6 we will discuss how uncertainties in the WIMP distribution affect these signals.

\subsection{Energy dependence}

The shape of the energy spectrum depends on both the WIMP mass and the mass of the target nuclei. This can be seen, following Lewin and Smith 65 , by assuming a standard Maxwellian velocity distribution, eq. (3), and, initially, neglecting the Earth's velocity and the Galactic escape speed. This allows the time averaged energy spectrum to be written as

$$
\frac{\mathrm{d} R}{\mathrm{~d} E}(E)=\left(\frac{\mathrm{d} R}{\mathrm{~d} E}\right)_{0} \exp \left(-\frac{E}{E_{\mathrm{R}}}\right) F^{2}(E),
$$

where $(\mathrm{d} R / \mathrm{d} E)_{0}$ is the event rate in the $E \rightarrow 0 \mathrm{keV}$ limit, and $E_{\mathrm{R}}$, the characteristic energy scale, is given by

$$
E_{\mathrm{R}}=\frac{2 \mu_{\mathrm{A} \chi}^{2} v_{c}^{2}}{m_{\mathrm{A}}} .
$$

When the Earth's velocity and the Galactic escape speed are taken into account eq. (21) is still a reasonable approximation to the event rate if $(\mathrm{d} R / \mathrm{d} E)_{0}$ and $E_{\mathrm{R}}$ are both multiplied by constants of order unity. If $m_{\chi} \ll m_{\mathrm{A}}, E_{\mathrm{R}} \propto m_{\chi}^{2}$, while if $m_{\chi} \gg m_{\mathrm{A}}, E_{\mathrm{R}}$ is independent of the WIMP mass. This indicates that the WIMP mass can be determined from the energy spectrum, provided it is not significantly larger than the mass of the target nuclei $76 / 77 / 78$. Furthermore measuring consistent spectra for two different target nuclei could in principle confirm the WIMP origin of these spectra (e.g. Ref. 79). This is sometimes referred to as the 'materials signal'. 


\subsection{Annual modulation}

Due to the Earth's orbit about the Sun, the net velocity of the lab with respect to the Galactic rest frame varies annually. The net speed is largest in the Summer and hence there are more high speed WIMPs, and less low speed WIMPs, in the lab frame. This produces an annual modulation of the event rate $6[80$. The differential event rate peaks in Winter for small recoil energies and in Summer for larger recoil energies 81. The energy at which the annual modulation changes phase is often referred to as the 'crossing energy'. Its value depends on the WIMP and target masses, and hence could be used to determine the WIMP mass 82 .

Since the Earth's orbital speed is significantly smaller than the Sun's circular speed the amplitude of the modulation is small and, to a first approximation, the differential event rate can, for the standard halo model, be written approximately as a Taylor series:

$$
\frac{\mathrm{d} R}{\mathrm{~d} E}(E, t) \approx \frac{\mathrm{d} R}{\mathrm{~d} E}(E)[1+\Delta(E) \cos \alpha(t)],
$$

where $\alpha(t)=2 \pi\left(t-t_{0}\right) / T, T=1$ year and $t_{0} \sim 150$ days. For the standard halo model the fractional amplitude of the modulation is approximately given by 83

$$
\Delta(E) \approx \begin{cases}-0.034\left(1-\frac{x^{2}}{x_{\mathrm{p}}^{2}}\right) & x<x_{\mathrm{p}} \\ 0.014\left(\frac{x}{x_{\mathrm{p}}}-1\right)\left(\frac{x}{x_{\mathrm{p}}}+3.7\right) & x_{\mathrm{p}}<x \lesssim z\end{cases}
$$

where $x=v_{\min } / v_{\mathrm{c}}, x_{\mathrm{p}}=0.89$ is the value of $x$ at which the sign of the modulation reverses and $z=v_{\text {esc }} / v_{\mathrm{c}}$. For $v_{\min } \gtrsim v_{\text {esc }}$, in the extreme tail of the speed distribution, the shape of the modulation is non-sinusoidal.

For very small energies $\Delta(E)$ is negative (i.e. the maximum occurs in December rather than June). As $E$ is increased, $|\Delta(E)|$ initially decreases to zero at which point the phase of the annual modulation changes so that the maximum occurs in Summer. As $E$ is increased further the fractional amplitude continues increasing. This is potentially misleading however, as the mean event rate, and hence the raw amplitude, becomes very small at large $E$. After the phase change on increasing $E$ further the raw amplitude increases to a local maximum before decreasing again and tending to zero. For the SHM, for measurable energies, the amplitude of the modulation lies in the range $1-10 \%$.

\subsection{Direction dependence}

Our motion with respect to the Galactic rest frame also produces a direction dependence of the event rate. The WIMP flux in the lab frame is peaked in the direction of motion of the Sun (towards the constellation CYGNUS), and hence the recoil spectrum is, for most energies $\mathrm{C}$, peaked in the direction opposite to this 86 . This

${ }^{\mathrm{c}}$ At low energies the maximum recoil rate is in a ring around the average WIMP arrival direction 8485. 
directional signal is far larger than the annual modulation; the event rate in the backward direction is several times larger than that in the forward direction $\frac{86}{}$. A detector which can measure the recoil directions is required to detect this signal (see Ref. 87 for an overview of the status of directional detection experiments).

The full direction dependence of the event rate is most compactly written in terms of the radon transform of the WIMP velocity distribution 88

$$
\frac{\mathrm{d} R}{\mathrm{~d} E \mathrm{~d} \Omega}=\frac{\rho_{0} \sigma_{\mathrm{p}} A^{2}}{4 \pi \mu_{\mathrm{p} \chi}^{2} m_{\chi}} F^{2}(E) \hat{f}\left(v_{\min }, \hat{\mathbf{q}}\right)
$$

where $\mathrm{d} \Omega=\mathrm{d} \phi \mathrm{d} \cos \gamma, \hat{\mathbf{q}}$ is the recoil direction and $\hat{f}\left(v_{\min }, \hat{\mathbf{q}}\right)$ is the 3 -dimensional Radon transform of the WIMP velocity distribution

$$
\hat{f}\left(v_{\min }, \hat{\mathbf{q}}\right)=\int \delta\left(\mathbf{v} \cdot \hat{\mathbf{q}}-v_{\min }\right) f(\mathbf{v}) \mathrm{d}^{3} \mathbf{v} .
$$

Geometrically the Radon transform, $\hat{f}\left(v_{\min }, \hat{\mathbf{q}}\right)$, is the integral of the function $f(\mathbf{v})$ on a plane orthogonal to the direction $\hat{\mathbf{q}}$ at a distance $v_{\min }$ from the origin. See Ref. 89 for an alternative, but equivalent, expression.

While the directional recoil rate depends on both of the angles which specify a given direction, the strongest signal is the differential of the event rate with respect to the angle between the recoil and the direction of solar motion, $\gamma, \underline{8690}$

$$
\frac{\mathrm{d}^{2} R}{\mathrm{~d} E \mathrm{~d} \cos \gamma}=\frac{\rho_{0} \sigma_{\mathrm{p}}}{4 \pi \mu_{\mathrm{p} \chi} m_{\chi}} A^{2} F^{2}(E) \int_{0}^{2 \pi} \hat{f}\left(v_{\min }, \hat{\mathbf{q}}\right) \mathrm{d} \phi
$$

For the standard Maxwellian velocity distribution 8690

$$
\frac{\mathrm{d}^{2} R}{\mathrm{~d} E \mathrm{~d} \cos \gamma}=\frac{C(\chi, A) \rho_{0}}{\sqrt{\pi} v_{\mathrm{c}}} \exp \left[-\left(\frac{\left(v_{\mathrm{e}}^{\mathrm{orb}, \mathrm{p}}+v_{\mathrm{c}}\right) \cos \gamma-v_{\min }}{v_{\mathrm{c}}}\right)^{2}\right]
$$

where $v_{\mathrm{e}}^{\mathrm{orb}, \mathrm{p}}$ is the component of the Earth's velocity parallel to the direction of Solar motion.

An ideal detector capable of measuring the nuclear recoil vectors (including their senses, $+\mathbf{q}$ versus $-\mathbf{q}$ ) in 3-dimensions, with good angular resolution, could reject isotropy of WIMP recoils with only of order 10 events 9189/92. Most, but not all, backgrounds would produce an isotropic Galactic recoil distribution (due to the complicated motion of the Earth with respect to the Galactic rest frame). An anisotropic Galactic recoil distribution would therefore provide strong, but not conclusive, evidence for a Galactic origin of the recoils. Roughly 30 events would be required for an ideal detector to confirm that the peak recoil direction coincides with the inverse of the direction of Solar motion, hence confirming the Galactic origin of the recoil events 9394 .

\section{Consequences}

In this section we will discuss how the uncertainties described in Secs. 3 and 4 affect the signals expected in direct detection experiments. 


\subsection{Energy dependence}

Since the normalisation of the event rate is directly proportional to the product of the cross-section, $\sigma_{\mathrm{p}}$, and the local density, $\rho_{0}$, the uncertainty in $\rho_{0}$ leads directly to an uncertainty in measurements of, or constraints on, $\sigma_{\mathrm{p}}$. Since the density is not expected to vary on mpc scales, the uncertainty in $\sigma_{\mathrm{p}}$ is the same for all direct detection experiments, and does not affect comparisons of e.g. the exclusion limits from different experiments. It does however affect comparisons with collider and other constraints on $\sigma_{\mathrm{p}}$. Furthermore it can significantly bias the determination of the WIMP mass 95 .

The characteristic energy scale, $E_{\mathrm{R}}$, given by eq. (22), depends on the local circular speed. Therefore the uncertainty in the local circular speed, $v_{\mathrm{c}}$, leads to a $\mathcal{O}(10 \%)$ uncertainty in the differential event rate, and hence exclusion limits 6896 . The nature of the change in the exclusion limits is similar, but not identical, for different experiments 1868 . It will also lead to a bias in determinations of the WIMP

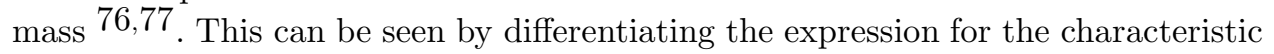
energy, eq. (22),

$$
\frac{\Delta m_{\chi}}{m_{\chi}} \sim-\left[1+\left(\frac{m_{\chi}}{m_{\mathrm{A}}}\right)\right] \frac{\Delta v_{\mathrm{c}}}{v_{\mathrm{c}}} .
$$

Since the differential event rate is given by an integral over the velocity distribution the differential event rate depends only weakly on the detailed form of the velocity distribution 9798 . Consequently the resulting uncertainty in exclusion

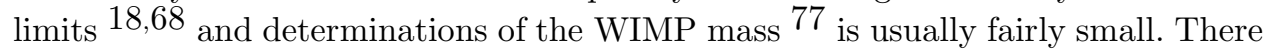
are, however, some exceptions to this statement. Firstly if the WIMP is light and/or the experimental energy threshold is high compared with the characteristic energy, then only the high speed tail of the speed distribution is probed. In this case the uncertainties in its shape can have a significant effect on the expected energy spectrum and hence exclusions limits or allowed regions 6816. Secondly if there is a dark disc there will be an additional population of low speed WIMPs. If the dark disc density is sufficiently high and its velocity dispersion sufficiently small this will significantly change the energy spectrum and hence exclusion limits and mass determinations 96 . The size of these changes depends on the properties of the dark disc, which are currently uncertain 48 151. A WIMP stream, with sufficiently high density, would add a sloping step to the differential event rate 75 .

\subsection{Annual modulation}

The annual modulation arises from the, relatively small, shift in the speed distribution in the lab frame over the course of the year. It is therefore far more sensitive to the speed distribution than the time averaged energy spectrum. Both the amplitude and phase of the modulation, and hence the regions of parameter space compatible with an observed signal, can vary significantly (e.g. Refs. 99, 100, 101, 102, 103, 104, 105, 106, 74, 96, 107). 
The uncertainty in the value of $v_{\mathrm{c}}$ can change the amplitude by a factor of order unity, while the uncertainty in the shape of the halo velocity distribution changes the amplitude by $\sim \mathcal{O}(10 \%)$ 10296. A high density dark disc could change the annual modulation signal significantly, producing a 2nd, low energy, maximum in the amplitude of the modulation and changing the phase significantly 96 . For a WIMP stream the position and height of the step in the energy spectrum produced would vary annually (e.g. Ref. 83).

\subsection{Direction dependence}

The detailed direction dependence of the event rate is sensitive to the velocity distribution, however the anisotropy is robust 918992. The number of events required to detect anisotropy or to demonstrate that the median inverse recoil direction coincides with the direction of solar motion only vary by of order $10 \% \underline{91|89| 92 \mid 94}$.

The features in the high speed tail can cause the median inverse direction of high energy recoils to deviate from the direction of solar motion $\underline{8}$. A stream of WIMPs would produce recoils which are strongly peaked in the opposite direction 70 . In the long term, studying the median direction of high energy recoils could allow high speed features to be detected and the formation history of the Milky Way probed.

\section{How to handle the uncertainties?}

\subsection{Astrophysics independent methods}

It is possible to carry out an 'astrophysics independent' comparison of data from multiple experiments. This effectively involves making comparisons of the energy spectra, so that the integral over the velocity distribution, $g\left(v_{\min }\right)$ defined in eq. (19), cancels. For instance, with data from two different experiments using two different targets, the WIMP mass can in principle be determined, without any assumptions about $f(\mathbf{v})$, by taking moments of the energy spectra 78 . However this method leads to a systematic underestimate of the WIMP mass if it is comparable to or larger than the mass of the target nuclei.

Ref. 108, see also Ref. 109, showed how the differential event rates in two experiments, ' 1 ' and ' 2 ' with target nuclei with mass numbers $A_{1}$ and $A_{2}$ are related. If the first experiment is sensitive to recoil energies in the range $\left[E_{1}^{\text {low }}, E_{1}^{\text {high }}\right]$ it probes $g\left(v_{\min }\right)$ for $v_{\text {min }}^{\text {low }} \leq v_{\text {min }} \leq v_{\text {min }}^{\text {high }}$, where $v_{\text {min }}$ is related to the recoil energy, WIMP mass and target nuclei mass by eq. (17). In experiment 2 these values of $v_{\text {min }}$ correspond to recoils in the range

$$
\left[E_{2}^{\text {low }}, E_{2}^{\mathrm{high}}\right]=\frac{\mu_{A 2 \chi}^{2} m_{A 1}}{\mu_{A 1 \chi}^{2} m_{A 2}}\left[E_{1}^{\text {low }}, E_{1}^{\mathrm{high}}\right]
$$

For recoil energies in this range the differential event rates in the two experiments 
are then related by

$$
\frac{\mathrm{d} R_{2}}{\mathrm{~d} E}\left(E_{2}\right)=\frac{C_{\chi, A_{2}}}{C_{\chi, A_{1}}} \frac{F_{2}^{2}\left(E_{2}\right)}{F_{1}^{2}\left(\frac{\mu_{A 1 \chi}^{2} m_{A 2}}{\mu_{A 2 \chi}^{2} m_{A 1}} E_{2}\right)} \frac{\mathrm{d} R_{1}}{\mathrm{~d} E}\left(\frac{\mu_{A 1 \chi}^{2} m_{A 2}}{\mu_{A 2 \chi}^{2} m_{A 1}} E_{2}\right) .
$$

This relation depends on the (unknown) WIMP mass, therefore the comparison has to be made separately for a range of $m_{\chi}$ values. This method has recently been extended to include annual modulation data 110 .

This approach has proved particularly useful 111112110 in comparing the event rate excesses and annual modulations found in the CoGeNT 113114, CRESST 115 and DAMA 116 experiments with exclusions limits from CDMS 117 and Xenon 118119. Ref. 110 found that the DAMA, CoGeNT and CRESST-II data are compatible which each other, but not CDMS and XENON, if the velocity distribution is very anisotropic (which leads to a large modulation fraction).

\subsection{Parameterizing the speed distribution}

The astrophysics independent methods are invaluable for assessing the compatibility of data from different experiments. However the WIMP mass is not a priori known, and the goal of WIMP direct detection experiments is not just to detect WIMPs, but to also measure their mass and cross-section. While there are uncertainties in the velocity distribution it is not completely unknown, and therefore does not need to be completely removed from the analysis.

Strigari and Trotta ${ }^{95}$ have shown how the WIMP properties can be constrained by a single future direct detection experiment when astronomical data (such as the kinematics of halo stars) are used to jointly constrain the WIMP properties and the parameters of a model for the Milky Way halo. They assumed an isotropic Maxwellian speed distribution characterised by the peak speed, $v_{0}$, and the escape speed, $v_{\text {esc }}$.

Peter 120121 has examined how data sets from multiple direct detection experiments could be used to jointly constrain a Maxwellian parametrisation of the WIMP speed distribution and the WIMP parameters (mass and cross-section). Pato et al. ${ }^{122}$ have taken a similar approach using the parameterization in eq. (15). Peter has recently extended this work by allowing the peak speed and circular speed to differ (as expected if the density profile is not $\rho(r) \propto r^{-2}$ ) and also considering an empirical speed distribution consisting of a five or ten bin step function 121 .

Combining data from multiple experiments with different targets significantly increases the accuracy with which the WIMP parameters can be measured $120 \mid 29$. However fixing the form of the speed distribution leads to biases in the WIMP properties, if the true speed distribution differs significantly from the parameterization assumed 121. With a suitable parameterization o the WIMP parameters and speed distribution can be jointly probed 121. The form of the optimal parameterization of the speed distribution is an open question. 


\section{Summary}

Direct detection event rate calculations often assume the standard halo model, with an isotropic Maxwellian velocity distribution with dispersion $\sigma=\sqrt{3 / 2} v_{\mathrm{c}}=$ $270 \mathrm{~km} \mathrm{~s}^{-1}$ and a local WIMP density $\rho_{0}=0.3 \mathrm{GeV} \mathrm{cm}^{-3}$. However it has long been realised $[6$ that uncertainties in the speed distribution will affect the signals expected in experiments.

We have discussed the standard halo model and other approaches to halo modelling, and the assumptions behind them. We then reviewed observational determinations of quantities that are relevant to direct detection experiments, namely the local dark matter density, the local circular speed (which is related to the velocity dispersion by the Jeans equations) and the local escape speed. While the statistical errors on these quantities are often small the systematic errors, from uncertainties in the modelling of the Milky Way, can be significantly larger.

Next we turned our attention to high resolution, dark matter only simulations of the formation of Milky Way-like halos in a cosmological context. They find velocity distributions that deviate significantly from the standard Maxwellian, and have features at high speeds. The effect of baryonic physics on the dark matter distribution is not yet well understood. Some recent simulations have found that a dark disc may be formed, however its properties (and even existence) are highly uncertain. Simulations resolve the dark matter distribution on scales many orders of magnitude larger than those probed by direct detection experiments. The latest results suggest that the ultra-local dark matter distribution is largely smooth, but some features may exist.

We then reviewed the resulting uncertainties in the energy, time and direction dependence of the energy spectrum, and hence constraints on, or measurements of, the WIMP parameters. The uncertainty in the local circular speed has a significant effect on the event rate and hence exclusion limits and determinations of the WIMP mass. The uncertainty in the local density leads directly to an uncertainty in measurements of, or constraints on, the cross-section. Only the high energy tail of the energy spectrum is particularly sensitive to the exact shape of the speed distribution. The annual modulation is far more sensitive to the shape of the velocity distribution; its amplitude and phase can change significantly. The anisotropy of the recoil rate is robust to changes in the velocity distribution, however high speed features can change the peak direction of high energy recoils, and hence provide a way of probing the formation of the Milky Way.

Finally we discussed techniques for handling the astrophysical uncertainties when analysing direct detection data. Astrophysics independent comparisons between different experiments can be made using the integral of the velocity distribution, $g\left(v_{\min }\right)$. This approach is extremely useful for assessing the compatibility of various experiments, but requires the WIMP mass as input. Parameterising the WIMP speed distribution, and using data from multiple experiments to jointly constrain the WIMP mass and cross-section and speed distribution is a promising 
approach, but the optimal form for the parameterisation is not yet known.

\section{Acknowledgments}

The author is supported by STFC. She is grateful to the organisers of, and participants in, the 'Dark matter underground and in the heavens' workshop (DMUH11) at CERN, where she had various useful discussions about the topics covered in this review. She is also grateful to Bradley Kavanagh for useful comments on a draft of this article.

\section{References}

1. G. Jungman, M. Kamionkowski and K. Griest, Phys. Rep. 267, 195 (1996).

2. G. Bertone, D. Hooper and J. Silk, Phys. Rep. 405, 279 (2005).

3. Particle Dark Matter, observations, models and searches, ed. G. Bertone, Cambridge University Press (2010).

4. M. W. Goodman and E. Witten, Phys. Rev. D 31, 3059 (1985).

5. B. Bertone, D. G. Cerdeno, M. Fornasa, R. Ruiz de Austri, C. Strege and R. Trotta, arXiv:1107.1715.

6. A. K. Drukier, K. Freese and D. N. Spergel, Phys. Rev. D 33, 3495 (1986).

7. J. F. Navarro, C. S. Frenk, and S. D. M. White, Astrophys. J. 462, 563 (1996), astro-ph/9508025.

8. M. Kuhlen et al., JCAP02(2010)030, arXiv:0912.2358.

9. J. Binney and S. Tremaine, Galactic Dynamics, Princeton University Press (2008).

10. F. J. Kerr and D. Lynden-Bell, Mon. Not. Roy. Astron. Soc. 221, 1023 (1986).

11. M. C. Smith et al., Mon. Not. Roy. Astron. Soc. 379, 755 (2007), astro-ph/0611671.

12. P. Ullio and M. Kamionkowski, JHEP03(2001)049, hep-ph/0006183.

13. J. D. Vergados and D. Owen, Astrophys. J 589, 17 (2003), astro-ph/0203293

14. J. D. Vergados and D. Owen, Phys. Rev. D 75043503 (2007), astro-ph/0603704.

15. R. Catena and P. Ullio, arXiv:1111.3556.

16. M. Lisanti, L. E. Strigari, J. G. Wacker and R. H. Wechsler, Phys. Rev. D 83, 023519 (2011), arXiv:1010.4300.

17. L. P. Osipkov, Pis'ma Astron. 55, 77 (1979); D. Merritt, Mon. Not. Roy. Astron. Soc. 90, 1027 (1984).

18. A. M. Green, Phys. Rev. D 66, 083003 (2002), astro-ph/0207366

19. N. W. Evans, C. M. Carollo and P. T. De Zeeuw, Mon. Not Roy. Astron. Soc. 318, 1131 (2000), astro-ph/0008156

20. P. Salucci, F. Nestri, G. Gentile and C. F. Martins, Astron. Astrophys. 523, 83 (2010), arXiv: 1003.3101

21. J. A. R. Caldwell and J. P. Ostriker, Astrophys. J, 251, 61 (1981).

22. E. I. Gates, G. Gyuk and M. S. Turner, Astrophys. J. 449, L123 (1995), astro-ph/9505039.

23. L. M. Widrow, B. Pym and J. Dubinski, Astrophys. J 679, 1239 (2008), arXiv:0801.3414.

24. R. Catena and P. Ullio, JCAP08(2010)004, arXiv:0907.0018.

25. J. F. Navvarro et al., Mon. Not. Roy. Astron. Soc. 349, 1039 (2004), astro-ph/0311231

26. A. W. Graham, D. Merritt, B. Moore, J. Diemand and B. Terzic, Astrophys. J 490, 493 (1997), astro-ph/0608613. 
27. M. Weber and W. de Boer, Astron. Astrophys 509, 25 (2010), arXiv:0910.4272

28. S. Garbari, J. I. Read and G. Lake, Mon. Not. Roy. Astron. Soc. 416, 2318 (2011), arXiv:1105.6339

29. M. Pato, O. Agertz, G. Bertone, B. Moore and R. Teyssier, Phys. Rev. D 82, 023531 (2010), arXiv: 1006.1322

30. O. Agertz, R. Teyssier and B. Moore, Mon. Not. Roy. Astron. Soc. 410, 1391 (2011), arXiv: 1004.0005

31. M. J. Reid et al., Astrophys. J 700, 137 (2009), arXiv:0902.3913

32. P. J. McMillan and J. J. Binney Mon. Not. Roy. Astron. Soc. 402, 934 (2010), arXiv:0907.4685

33. J. Bovy, D. W. Hogg and H. Rix, Astrophys. J 704, 1704 (2009), arXiv:0907.4523

34. P. J. T. Leonard and S. Tremaine, Astrophys. J 353, 486 (1990).

35. C. S. Kochanek, Astrophys. J 457, 228 (1996).

36. V. Springel et al., Mon. Not. Roy. Astron. Soc. 391, 1685 (2008), arXiv:0809.0898

37. J. Stadel et al., Mon. Not. Roy. Astron. Soc. 398, L21 (2009), arXiv:0808.2981

38. J. Diemand et al., Nature 454, 735 (2008), arXiv:0805.1244.

39. S. H. Hansen, B. Moore, M. Zemp and J. Stadel, JCAPA01(2006)014, astro-ph: 0505420

40. M. Fairbairn and T. Schwetz, JCAP01(2009)037, arXiv:0808.0704

41. M. Vogelsberger et al., Mon. Not. Roy. Astron. Soc. 395, 797 (2009), arXiv:0812.0362

42. C. Stasllis, J. Stat. Phys. 52, 479 (1988).

43. S. H. Hansen, D. Egli, L. Hollenstein and C. Salzmann, New. Astron. 10, 379 (2005), astro-ph/0407111

44. J. I. Read, G. Lake, O. Agertz and V. P. Debattista, Mon. Not. Roy. Astron. Soc. 389, 1041 (2008), arXiv:0803.2714

45. J. I. Read, L. Mayer, A. M. Brooks, F. Governato and G. Lake, Mon. Not. Roy. Astron. Soc. 397, 44 (2009), arXiv:0902.0009

46. F.-S. Ling, E. Nezri, E. Athanassoula and R. Teyssier, JCAP02(2010)012 arXiv:0909.2028

47. T. Bruch, J. Read, L. Baudis and G. Lake, Astrophys. J 696, 920 (2009), arXiv:0804.2896

48. C. W. Purcell, J. S. Bullock and M. Kaplinghat, Astrophys. J 703, 2275 (2009), arXiv:0906.5348

49. J. Holmberg and C. Flynn, Mon. Not. Roy. Astron. Soc. 313, 209 (2000), astro-ph/9812404

50. C. M. Bidin, G. Carraro, R. A. Mendez and W. F. van Altena, Astrophys. J. Lett. 724, L122 (2010), arXiv:1011.1289.

51. F.-S. Ling, Phys. Rev. D 82, 023534 (2010), arXiv:0911.2321

52. S. Kazantzidis et al., Astrophys. J 611, L73 (2004), astro-ph/0405189.

53. M. Vogelsberger and S. D. M. White, Mon. Not. Roy. Astron. Soc. 413, 1419 (2011), arXiv: 1002.3162

54. A. Schneider, L. M. Krauss and B. Moore, Phys. Rev. D 82, 063525 (2010), arXiv: 1004.5432

55. S. Hofmann, D. J. Schwarz and H. Stocker, Phys. Rev. D 64, 083507 (2001), astro-ph0104173

56. A. M. Green, S. Hofmann and D. Schwarz, Mon. Not. Roy. Astron. Soc. 353, L23 (2004), astro-ph/0309621

57. A. M. Green, S. Hofmann and D. Schwarz, JCAP08(2005)003, astro-ph0503387.

58. J. Diemand, B. Moore and J. Stadel, Nature 433, 289 (2005), astro-ph/0501589 
59. M. Kamionkowski and S. M. Koushiappas, Phys. Rev. D 77, 103509 (2008), arXiv:0801.3269.

60. D. S. M. Fantin, M. R. Merrifield and A. M. Green, Mon. Not. Roy. Astron. Soc. 390, 1055 (2008), arXiv:0808.1050.

61. N. Afshordi, R. Mohayee and E. Bertschinger, Phys. Rev. D 79, 083526 (2009), arXiv:0811.1582.

62. N. Afshordi, R. Mohayee and E. Bertschinger, Phys. Rev. D 82, 101301 (2010), arXiv:0911.0414.

63. M. Lisanti and D. Spergel, arXiv:1105.4166.

64. D. S. M. Fantin, A. M. Green and M. R. Merrifield, Mon. Not. Roy. Astron. Soc. 418, 2648 (2011), arXiv:1108.4411.

65. J. D. Lewin and P. F. Smith, Astropart. Phys. 6, 87 (1996).

66. D. Tucker-Smith and N. Weiner, Phys. Rev. D 64, 043502 (2001), hep-ph/0101138

67. J. March-Russell, C. McCabe and M. McCullough, JHEP05(2009)071, arXiv:0812.1931.

68. C. McCabe, Phys. Rev. D 82, 023530 (2010), arXiv:1005.0579.

69. K. Griest Phys. Rev. D 37, 2703 (1988).

70. M. S. Alenazi and P. Gondolo, Phys. Rev. D 74, 083518 (2006), astro-ph: 0608390.

71. R. Schoenrich, J. Binney and W. Dehnen, Mon. Not. Roy. Astron. Soc. 403, 1829 (2010), arXiv:0912.3693.

72. W. Dehnen and J. J. Binney, Mon. Not. Roy. Astron. Soc. 298, 387 (1998), astro-ph/9710077

73. R. M. Green, Spherical Astronomy, Cambridge University Press (1985).

74. N. Fornengo and S. Scopel, Phys. Let. B 576, 189 (2003), astro-ph/0301132

75. G. Gelmini and P. Gondolo, Phys. Rev. D 64, 023504 (2001) hep-ph/0012315

76. http://cosmology.berkeley.edu/inpac/CDMSCE_Jun06/Talks/200606CDMSCEmass.pdf

77. A. M. Green, JCAP08(2007)022, hep-ph/0703217 JCAP07(2008)005, arXiv:0805.1704.

78. C.-L. Shan and M. Drees, JCAP06(2008)012, arXiv:0803.4477

79. H. Kraus et al., Phys. Lett. B 610, 37 (2005).

80. K. Freese, J. A. Frieman and A. Gould, Phys. Rev. D 37, 3388 (1988).

81. J. R. Primack, D. Seckel and B. Sadoulet, Ann. Rev. Nucl. Part. Sci. 38, 751 (1988).

82. M. J. Lewis and K. Freese, Phys. Rev. D 70, 043501 (2004), astro-ph/0307190.

83. C. Savage, K. Freese and P. Gondolo, Phys. Rev. D 74, 043531 (2006), astro-ph/0607121.

84. N. Bozorgnia, G. B. Gelmini and P. Gondolo, Phys. Rev. D 84, 023516 (2011), arXiv:1101.2876

85. N. Bozorgnia, G. B. Gelmini and P. Gondolo, arXiv:1111.6361.

86. D. N. Spergel, Phys. Rev. D 37, 1353 (1998).

87. S. Ahlen et al., Int. J. Mod. Phys. A. 25, 1 (2010), arXiv:0911.0323.

88. P. Gondolo, Phys. Rev. D 66, 103513 (2002), astro-ph/0209110

89. C. J. Copi and L. M. Krauss, Phys. Rev. D 63, 043508 (2001), astro-ph/0009467.

90. M. S. Alenazi and P. Gondolo, Phys. Rev. D 77, 043532 (2008), arXiv:0712.0053.

91. C. J. Copi, J. Heo and L. M. Krauss, Phys. Lett. B 461, 43 (1999), astro-ph/990449.

92. B. Morgan, A. M. Green and N. J. C. Spooner, Phys. Rev. D 71, 103507 (2005), astro-ph/0408047.

93. J. Billard, F. Mayet, J. F. Macias-Perez and D. Santos, Phys. Lett. B 691, 156 (2010), arXiv:0911.04086.

94. A. M. Green and B. Morgan, Phys. Rev. D 81, 061301(R) (2010), arXiv:1002.2717 
95. L. E. Strigari and R. Trotta, JCAP11(2009)019, arXiv:0906.5361.

96. A. M. Green, JCAP10(2010)034, arXiv:1009.0916.

97. M. Kamionkowski and A. Kinkhabwala, Phys. Rev. D 57, 3256 (1998), hep-ph/9710337.

98. F. Donato, N. Fornengo and S. Scopel, Astropart. Phy. 9, 247 (1998)247, hep-ph: 9803295.

99. M. Brhlik and L. Roszkowski, Phys. Lett. B 464, 303 (1999), hep-ph/9903468

100. J . D. Vergados, Phys. Rev. Lett. 83, 3597 (1999); Phys. Rev. D 62, 023519 (2000), astro-ph/0001190.

101. P. Belli et. al., Phys. Rev. D 61, 023512 (2000).

102. A. M. Green, Phys. Rev. D 63, 043005 (2001), astro-ph/0008318.

103. J . D. Vergados, Phys. Rev. D 63, 062511 (2001).

104. Phys. Rev. D 68, 023004 (2003), astro-ph/0304446

105. R. Bernabei et al., Int. J. Mod. Phys. D 13, 2127 (2004), astro-ph/0501412

106. J. D. Vergados, S. H. Hansen and O. Host, Phys. Rev. D 77, 023509 (2008), arXiv:0711.4895.

107. S. Chaudhury, P. Bhattacharjee and R. Cowsik, JCAP09(2010)020, arXiv: 1006.5588 .

108. P. J. Fox, J. Liu and N. Weiner, Phys. Rev. D 83, 103514 (2011), arXiv:1011.1915.

109. P. J. Fox, G. D. Kribs and T. M. P. Tait, Phys. Rev. D 83, 034008 (2011), arXiv: 1011.1910

110. M. T. Frandsen, F. Kahlhoefer, C. McCabe, S. Sarkar and K. Schmidt-Hoberg, arXiv:1111.0292.

111. P. J. Fox, J. Kopp, M. Lisanti and N. Weiner, arXiv:1107.0717

112. C. McCabe, Phys. Rev. D 84, 043525 (2011), arXiv:1107.0741.

113. C. E. Aalseth et al., Phys. Rev. Lett. 106, 131301 (2011), arXiv:1002.4703;

114. C. E. Aalseth et al., Phys. Rev. Lett. 107, 141301 (2011), arXiv:1106.0650.

115. G. Angloher et al., arXiv:1109.0702.

116. R. Bernabei et al., Eur. Phys. C 56, 39 (2010) arXiv:1002.1028.

117. D. S. Akerib et al., Phys. Rev. D 82, 122004 (2010), arXiv:1010.4290

118. J. Angle et al., Phys. Rev. Lett. 107, 051301 (2011), arXiv:1104.3088

119. E. Aprile et al., Phys. Rev. Lett. 107, 131302 (2011), arXiv:1104.2549.

120. A. H. G. Peter, Phys. Rev. D 81, 087301 (2010), arXiv:0910.4765

121. A. H. G. Peter, Phys. Rev. D 83, 125029 (2011), arXiv:1103.5145

122. M. Pato et al., Phys. Rev. D 83083505 (2011), arXiv:1012.3458. 